\title{
Beobachtungen zur Rezeption des Euripides bei den Lexikographen*
}

\begin{abstract}
The reception of Euripides in Greek lexicography dating from the Imperial age to Late Antiquity is complex and has never been extensively investigated. The present paper intends to offer a preliminary sketch of this large potential research field, trying to identify trends in the methods of citation from Euripides in the surviving versions of lexica from that period. Entries in the lexica of Hesychius, Harpokration, Stephanus of Byzantium as well as in Orion's etymologicum are analysed to reveal common features. Generally speaking, quotations from Euripides's works are not taken by the lexicographers from a first hand reading of his plays, but rather from intermediate sources. Among them, the works by Didymus of Alexandria probably played a pivotal role. Citations of verses from Euripides's plays served mainly to illustrate the content of a given entry or a peculiar literary usage by means of a pertinent quotation. In the Atticist lexica, we encounter a more partial use of Euripides. Citations from his plays often serve to introduce or to support a particular meaning of a word with the objective of supporting or condemning its use in contemporary literary production. In this context, the most rigorous Atticists usually tend to criticise his linguistic usage.
\end{abstract}

\section{Einführung und Problemstellung}

Die Euripides-Rezeption in der lexikographischen Produktion aus der Kaiserzeit und Spätantike ist vielfältig und zum großen Teil noch zu erforschen. Lexika im Allgemeinen wurden meistens mit spezifischen Zielsetzungen untersucht, sei es als Quelle von Zitaten aus nicht mehr erhaltenen Werken dieses Klassikers oder als indirekte Zeugen für den Text oder die Interpretation problematischer Stellen der überlieferten Tragödien. Selten wurden aber die Motivation und die Zwecke erforscht, die die Lexikographen bewegten, Euripides heranzuziehen.

Diese Untersuchung ist zudem dadurch erschwert, das noch kein ausführliches Repertorium der Zitate aus den erhaltenen Tragödien des Euripides in der lexikographischen Produktion vorhanden ist: Dies wurde mit aller Deutlichkeit von William Spencer Barrett in der Einleitung zu seiner Ausgabe des Hippolytos bemängelt: „No exhaustive list of these quotations [scil. „in medieval lexica and scholia“] exists. The fullest is in Kirchhoff (mostly taken over, complete with false references, via Monk

\footnotetext{
* Gedankt sei den Teilnehmern der Tagung für ihre Anmerkungen und Anregungen zum Vortrag, der hier in einer leicht überarbeiteten Fassung erscheint, sowie Klaus Alpers, Christian Brockmann, Daniel Deckers und Renzo Tosi für ihre sehr nützlichen Kommentare.
} 
from Valckenaer). “1 Nur für die Fragmente steht das Quellenregister des fünften Bands der Tragicorum Graecorum Fragmenta von Richard Kannicht zur Verfügung. ${ }^{2}$

In dem vorliegenden Beitrag werden nur wenige Aspekte in den Blick genommen, um eine erste Sichtung dieser umfangreichen Problematik vorzunehmen. Als Basis dieser Untersuchung dienen diejenigen lexikographischen Stellen, an denen Euripides namentlich erwähnt wird. Zitate aus noch überlieferten Tragödien werden in der Regel bevorzugt, um die lexikographischen Stellen mit dem vollständigen euripideischen Kontext vergleichen zu können. Denn auf diese Weise können allgemeine Tendenzen im Zitierstil unterschiedlicher Lexikographen besser evaluiert werden als bei Zitaten, bei denen uns der Kontext des Dramas fehlt. Die durch diese Methode gewonnenen Ergebnisse könnten danach analog auf die nur indirekt überlieferten Texte übertragen werden.

Die Untersuchung literarischer Zitate in lexikographischen Texten setzt außerdem die Erkenntnis voraus, dass die überlieferten Versionen der Lexika keineswegs den ursprünglichen Textfassungen entsprechen. Viele Zitate sind im Laufe der Überlieferungsgeschichte jedes Lexikons weggelassen worden, viele wiederum verkürzt bzw. willkürlich oder bewusst modifiziert worden. Erforderlich ist deshalb eine akkurate überlieferungsgeschichtliche und quellenkritische Analyse jedes Lexikons und quasi jeder Glosse, um die einzelnen Erwähnungen des Euripides bestmöglich verstehen zu können. ${ }^{3}$

\section{Euripides-Zitate bei Hesych}

Eine der primären und am häufigsten herangezogenen Quellen für Euripides-Zitate in der griechischen Lexikographie ist gewiss das Lexikon des Hesych, ${ }^{4}$ ein sehr umfangreiches Werk, das zwischen dem 5. und 6. Jh. n.Chr. verfasst wurde. Die von Hesych gesammelten Materialien stammen aus unterschiedlichen gelehrten Quellen, wie der Lexikograph selbst in seinem Widmungsbrief angibt. ${ }^{5}$ Wie schon Ulrich von Wilamowitz-Moellendorff in seiner Einleitung in die griechische Tragödie feststellten konnte, stammen die meisten Euripides-Zitate in diesem Lexikon sehr wahrscheinlich aus den Werken von Didymos, sei es direkt oder vermittels einer oder mehrerer Zwischenquelle(n). ${ }^{6}$ In der erhaltenen Fassung des Lexikons wird Euripides 78 Mal namentlich zitiert; ${ }^{7}$ in mehreren anderen Glossen ist sein Name wohl beim Kürzungs-

1 Barrett 1964, 83 Anm. 4.

2 Kannicht 2004, II 1045-1088 (Index fontium).

$3 \mathrm{Zu}$ dieser Fragestellung vgl. u. a. Tosi 1988; Alpers 1990, $30 \mathrm{f}$.

4 Vgl. u. a. Latte 1953, VII-LI; Alpers 2005; Cunningham 2009; Matthaios 2015, $289 \mathrm{f}$.

5 Latte 1953, 1f. = Latte-Cunningham 2018, 1f. (vgl. auch Valente 2018).

6 Wilamowitz 1921, $163-165$.

7 So das Resultat einer Recherche beim TLG online (Stand: 30.6.2017); vgl. auch das Autorenregister bei Schmidt 1868, 94-100. 
und Überarbeitungsprozess verlorengegangen. In der Regel dienen Euripides-Zitate als Belegstellen für die erklärten Begriffe, wie z. B. in folgender Glosse:

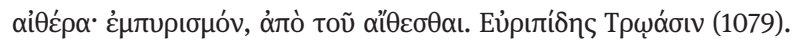

Äther ( $\left.\alpha^{i} \theta \varepsilon \dot{\rho} \rho \alpha\right)$ : In-Brand-Setzen, Brand von „brennen“ ( $\left.\alpha^{\prime} \theta \varepsilon \sigma \theta \alpha \mathrm{l}\right)$. Euripides in den Troerinnen (1079). (Hesych. $\alpha 1855$ Latte-Cuninngham)

Die etymologische Erklärung des Begriffes aiӨń (Äther) wird durch die Erwähnung eines Passus der Troerinnen von Euripides belegt. Im Text des Hesychlexikons ist kein Zitat überliefert, aber die genaue Textstelle lässt sich leicht identifizieren:

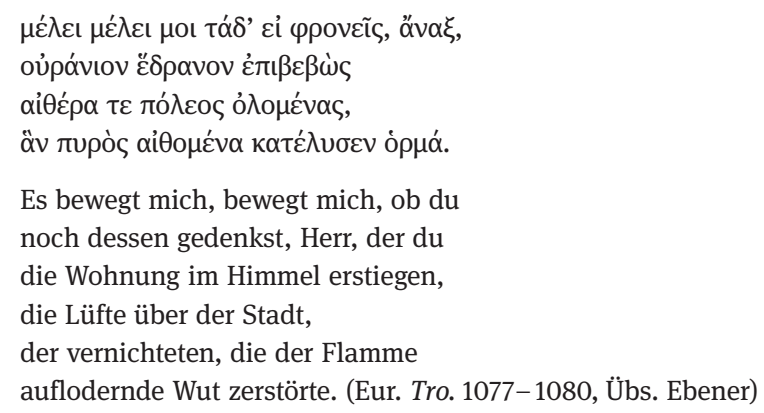

Nach einer nicht unüblichen Methode in der antiken Gelehrsamkeit wurde die Erklärung anhand des Kontexts der zitierten Textstelle zusammengestellt. Die Hesychglosse wird auch im Testimonienapparat der Teubner-Ausgabe von Werner Biehl angegeben. ${ }^{8}$ In seinem Kommentar schreibt er zu dieser Stelle Folgendes:

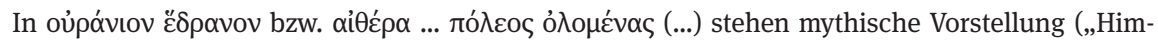
mel") und auf philosophischer bzw. naturwissenschaftlicher Lehre beruhende Auffassung („Äther“ als Ergebnis eines Verbrennungsprozesses), durch parataktische Partikel ( $\tau \varepsilon$ ) verbunden, nebeneinander. Die etymologische Anspielung (1079f. ai $\theta \varepsilon^{\prime} \rho \alpha$... ai $\theta$ ó $\mu \varepsilon v \alpha$ ) kann vermutlich als ein Hinweis verstanden werden, daß der Chor auch weiterhin an eine Verbundenheit des Zeus mit Troja denkt bzw. eine solche mit Bitterkeit vermißt. ${ }^{9}$

Was der moderne Herausgeber und Kommentator thematisiert, wurde schon von einem alten Lexikographen angedeutet, mit dem Ziel, die Bedeutung eines poetischphilosophischen Wortes genau zu erklären.

Ein Vergleich mit dem entsprechenden byzantinischen Scholion zu diesem Vers zeigt, dass die Hesychglosse letztlich von dem Werk des alexandrinischen Gelehrten Didymos (1. Jh. v.Chr.) stammt: ${ }^{10}$

\footnotetext{
8 Biehl 1970, 58.

9 Biehl 1989, 387.

10 Das Scholion wird jetzt auch im Apparat zur Hesychglosse bei Latte-Cunningham 2018, 87 erwähnt.
} 


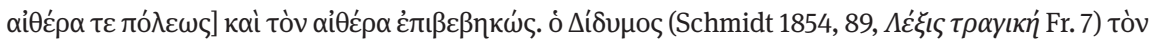

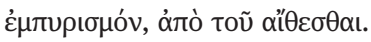

Und den Äther über der Stadt] und den Äther hat er erstiegen. Didymos setzt den Äther mit dem Brand gleich und leitet ihn von dem Verb „brennen“ ( $\alpha$ ï $\theta \varepsilon \sigma \theta \alpha \iota)$ ab. (Schol. Eur. Tro. 1079 Schwartz)

Im Lexikon des Patriarchen Photios finden wir außerdem fast dieselbe Glosse wie im Hesychlexikon:

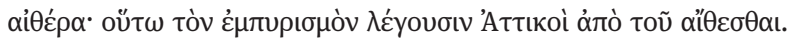

Äther: So nennen die Attiker den Brand von „brennen“ ( $\alpha i ̈ \theta \varepsilon \sigma \theta \alpha \mathrm{l})$. (Phot. Lex. $\alpha 570$ Theodoridis)

Photios und Hesych benutzen eine gemeinsame Zwischenquelle, und zwar das Lexikon des Diogenian, der seinerseits unter seinen Quellen auch Didymos hatte. Deshalb ist zu vermuten, dass Photios und Hesych dieses Euripides-Zitat nicht direkt von dem alexandrinischen Gelehrten übernommen haben, sondern eher aus ihrer gemeinsamen Zwischenquelle, nämlich Diogenian. ${ }^{11}$ Die - attizistische - Angabe oi

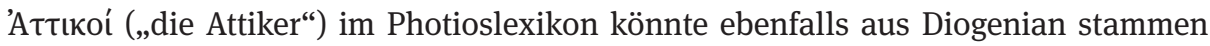
und im Hesychlexikon ausgelassen worden sein; umgekehrt dürfte die Erwähnung von Euripides im Photioslexikon weggefallen sein.

Hinsichtlich der Euripides-Zitate im Lexikon des Hesych lässt sich im Allgemeinen die Einsicht von Richard Kannicht in der Einleitung zu seiner kommentierten Ausgabe der Helena auch auf die anderen Dramen ausdehnen:

Die letzten Spuren dieses Vorgangs [d.h. der lexikographischen Aufarbeitung euripideischer Tragödien] sind für uns im Lexikon des Hesychios (5./6. Jh. n.Chr.) als dem spätantiken Endprodukt eines Prozesses stetiger Verdünnung und Verarmung des ursprünglich reichen Materials erkennbar. Für die Sprache der Tragödie scheint die Quelle Pamphylos $\rightarrow$ Diogenian $\rightarrow$ Hesychs die

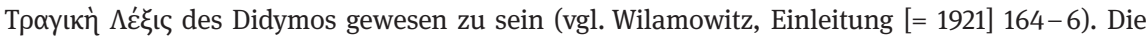
Tragikerglossen Hesychs werden also in der Regel auf die Sammlung des Didymos und häufig über Didymos auf die Hypomnemata der frühen Alexandriner zurückgehen. Ihre Identifizierung ist jedoch grundsätzlich immer dann unsicher, wenn der Verdünnungsprozeß zum Verlust der Quellenangabe geführt hat. ${ }^{12}$

Mit gutem Recht mahnt Kannicht außerdem zur Vorsicht, anonyme Hesychglossen als euripideisch zu bezeichnen, wenn der Name des Dichters fehlt. Ein ähnliches vorsichtiges Verfahren hatte schon Eduard Fraenkel in der Einleitung zu seiner kommentierten Ausgabe des Agamennon von Aischylos für sich beansprucht. ${ }^{13}$

11 Diese Vermutung wird auch durch eine Glosse im Etymologicum Magnum $(33,9)$ bestätigt, die schon Latte (1953, 67, vgl. auch Latte-Cunningham 2018, 87), Biehl $(1970,58)$ und Theodoridis $(1982,66)$

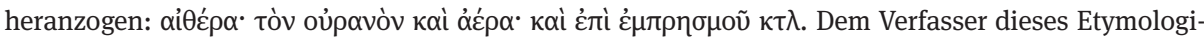
kons war eine Kopie des Lexikons von Diogenian zugänglich (s. dazu u.a. Reitzenstein 1897, 251f.). 12 Kannicht 1969, I 90.

13 Fraenkel 1950, 11. 


\section{Euripides-Zitate in den Rednerlexika und bei Stephanos von Byzanz}

Betrachtet man andere Arten von Lexika, lassen sich ähnliche Tendenzen entdecken. In den Rednerlexika sowie im geographischen Lexikon des Stephanos von Byzanz scheint Euripides im Allgemeinen meist aus grammatikalischen bzw. lexikographischen Zwischenquellen herangezogen worden zu sein. Zwei Beispiele können als Beleg dafür gelten.

\subsection{Das Rednerlexikon des Harpokration}

Das erste Beispiel entstammt dem aus dem 2./3. Jh. n. Chr. stammenden Lexikon des Harpokration ${ }^{14} \mathrm{zu}$ den zehn Rednern:

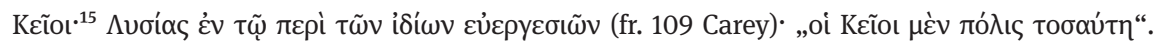

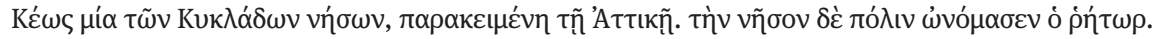

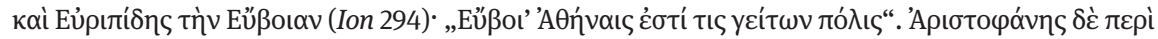

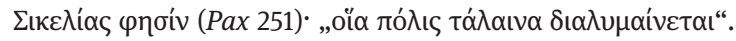

Keïer: Lysias in der Rede Über die privaten Verdienste (fr. 109 Carey): „Die Keïer sind eine so große Stadt.“ Keos ist eine Insel der Kykladen, die vor Attika liegt. Der Redner nannte die Insel „Stadt“. Auch Euripides (Ion 294) nannte Euboia „Stadt“: „Euboia ist eine Nachbarstadt von Athen“. Seinerseits sagt Aristophanes (Pax 251) in Bezug auf Sizilien: „Was für eine Stadt elend zerstört wird“. (Harp. 173,4-10 Dindorf $=\kappa 34$ Keaney)

Hier wird zunächst ein Vers aus dem euripideischen Ion zitiert:

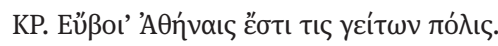

Euboia ist eine Nachbarstadt von Athen. (Eur. Ion 294)

dann eine Stelle aus Aristophanes’ Frieden:

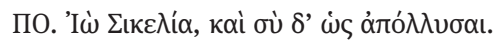

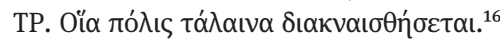

(Polemos) O weh, Sizilien, wie auch du zugrunde gehst!

(Trygaios) Was für eine Stadt elend zerrieben werden wird. (Ar. Pax 250f.)

$14 \mathrm{Zu}$ Harpokration vgl. Matthaios 2015, 284 mit weiteren Literaturhinweisen.

15 Aus der Epitome stammt die Glosse der „erweiterten Synagoge“, die bei Phot. Lex. $\mathrm{741}$ Theod. und Suid. $\kappa 1690$ Adler zu lesen ist.

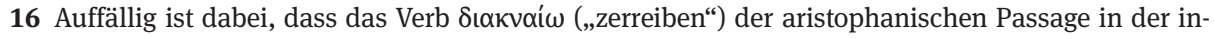
direkten Überlieferung durch ein Synonym $(\delta\llcorner\alpha \lambda \nu \mu \alpha i ́ v \omega)$ ersetzt wurde. 
Ziel beider Zitate ist es, ein Pendant für die auffällige Bezeichnung der Insel Keos als Polis in einer heute verschollenen Rede des Lysias zu finden. Dieselbe Erklärung mit demselben Zitat aus dem Ion von Euripides lässt sich u. a. auch in einem Scholion zum zitierten Passus von Aristophanes' Frieden finden, in dem außerdem Homer für diese Gewohnheit herangezogen wird:

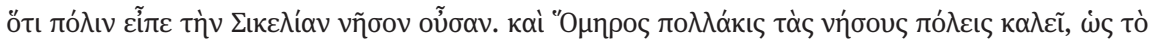

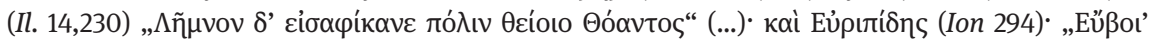

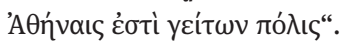

Aristophanes nannte Sizilien eine Stadt, obwohl es doch eine Insel ist. Auch Homer $(I l .14,230)$ nennt oft die Inseln Städte, wie zum Beispiel in dem Vers: „Lemnos erreichte sie dann, die Stadt des göttlichen Thoas“ (Übs. Voss) (...); und Euripides (Ion 294): „Euboia ist eine Nachbarstadt von Athen“. (Schol. Ar. Pax 251a Holwerda)

Die Koinzidenz zwischen dem Scholion und dem Lexikon des Harpokration weist darauf hin, dass diese gelehrten Materialien auch in diesem Fall aller Wahrscheinlichkeit nach von Didymos stammen, denn sein Werk stellt eine der wesentlichen Grundlagen der Aristophanes-Scholien dar. ${ }^{17}$ Aus derselben Quelle stammt wohl auch eine Hesych-Glosse zum Begriff $\pi$ ó̀ıc: ${ }^{18}$

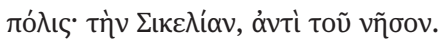

Stadt: Sizilien, anstelle von Insel. (Hesych. $\pi 2787$ Hansen)

Auch für die hier behandelten lexikographischen Stellen lässt sich keine direkte Benutzung des Euripides-Textes erkennen.

\subsection{Das geographische Lexikon des Stephanos von Byzanz}

Das zweite Beispiel entstammt dem geographischen Lexikon des Stephanos von Byzanz (Anfang des 6. Jh.). In den Ethnika werden Euripides-Zitate als literarische Belege für die besprochenen Ortsangaben angeführt. Die meisten davon lassen sich eher als Zitate aus mindestens zweiter Hand einordnen, wie z.B. dasjenige in der folgenden Glosse:

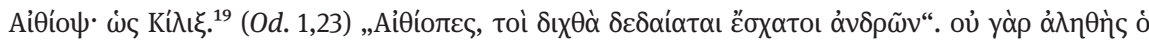

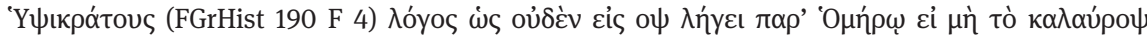

17 Zu Didymos als Quelle der Aristophanes-Scholien vgl. u. a. Dunbar 1995, 37-42; Braswell 2013, 44 f., $63 \mathrm{f}$.

18 Vgl. auch Schol. Il. 14,230 Erbse, Poll. 9,27 Bethe. - Hätten wir aber keinen weiteren lexikographischen Beleg, wäre eine solche Zuweisung ohne Weiteres gewiss nicht erlaubt.

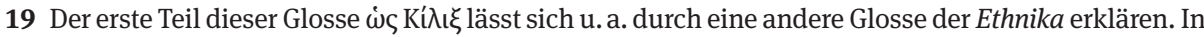

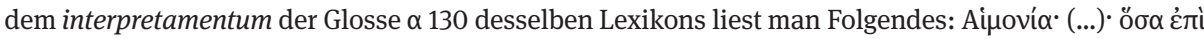




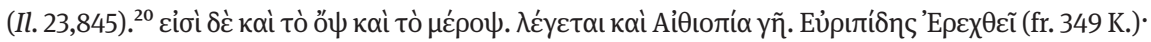

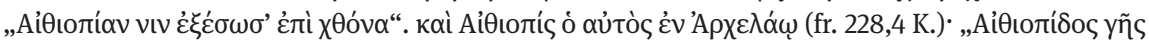

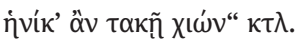

Aithiops, <gebildet> wie Kilix. (Od.1,23) „Die Aithiopen, die am äussersten Rand der Menschheit in zwei Gruppen geteilt sind“. Denn nicht zutreffend ist die Behauptung des Hypsikrates (FGrHist

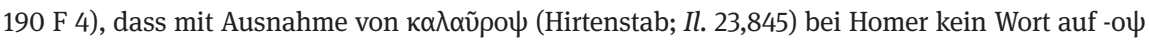

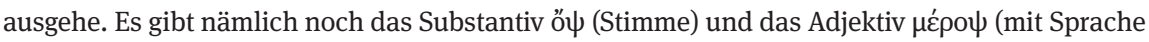
begabt). Man spricht auch vom aithiopischen Land. <So sagt> Euripides im Erechtheus (fr. 349 Kannicht = fr. 2 Jouan/van Looy): „Ihn rettete ich auf aithiopischen Boden“. Und derselbe <verwendet> im Archelaos (fr. 228,4 Kannicht = fr. 1a, 4 Jouan/van Looy) <das Femininum > aithiopidische „... der aithiopidischen Erde, wenn der Schnee zu schmelzen beginnt“ [usw.]. (Steph. Byz. $\alpha 124$ Billerbeck, Übs. Billerbeck)

Das Ziel, das durch die Zitate aus Euripides erreicht werden soll, liegt zuvorderst nicht auf dem poetischen Text an sich, denn Euripides wird nur als - eher indirekte - Quelle topographischer Begriffe und selten belegter Wortformen herangezogen, und zwar

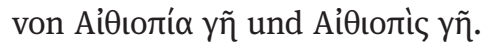

\section{Euripides im Etymologikon des Orion}

Einen grammatikalischen Ursprung zeigen auch die Euripides-Zitate im Etymologikon des Orion, eines Grammatikers aus dem 5. Jh. n.Chr. ${ }^{21}$ Eine Auswertung solcher Zitate ist aber dadurch erschwert, dass noch keine kritische Ausgabe des Textes von Orion vorhanden ist. Dabei handelt es sich um das erste Etymologikon, d. h. um ein Lexikon, dessen Schwerpunkt auf Untersuchungen zu den richtigen Bedeutungen der Wörter anhand der Analyse ihrer Herkunft liegt. Die Überlieferungsgeschichte dieses Textes ist zum größten Teil noch zu klären; ${ }^{22}$ außer den noch erhaltenen Manuskripten des Lexikons sind auch spätere lexikographische Werke unverzichtbar für die Herstellung des Textes des Orion, weil sie ihn noch in einer ausführlicheren Fassung lesen konnten: Hier ist besonders an die byzantinischen Etymologika zu denken. ${ }^{23}$ An dieser Stelle sei nur eine Glosse aus dem genannten Lexikon besprochen, um diese problematische Lage $\mathrm{zu}$ verdeutlichen:

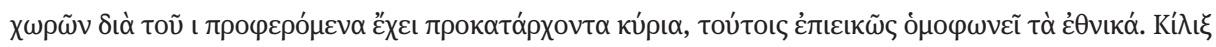

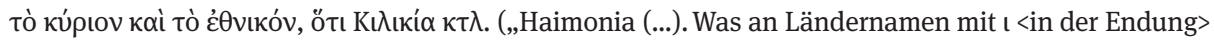
zitiert wird und auf Personennamen zurückgeht, hat in der Regel Ethnika, welche mit ebendiesen $<$ Personennamen> übereinstimmen: Kílı $\xi$ ist sowohl der Personenname als auch das Ethnikon (Kilike), weil <das Land $>$ Kilikia <heisst > usw. “, Übs. Billerbeck).

$20 \mathrm{Zu}$ dieser Stelle vgl. bes. Billerbeck u. Neumann-Hartmann 2017, 163.

21 Vgl. Matthaios 2015, $287 \mathrm{f}$. mit Literatur.

22 Vgl. Theodoridis 1976, 15-19, bes. 15 mit Anm. 2.

23 Vgl. Reitzenstein 1897, 243-248; Theodoridis 1976, 15 f. 


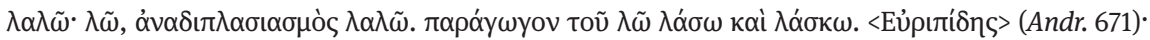

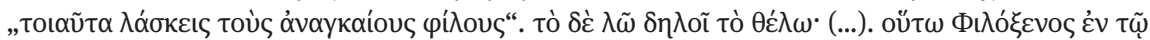

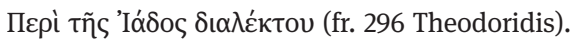

Schwatzen: Grundform $\lambda \tilde{\omega}$, mit Verdoppelung $\lambda \alpha \lambda \tilde{\omega}$. Abwandlungen von $\lambda \tilde{\omega}$ sind die Formen $\lambda \alpha \dot{\sigma} \sigma \omega$ und $\lambda \alpha ́ \sigma \kappa \omega$. Euripides (Andr. 671) sagt: „Du schmähst auf solche Weise die nächsten Freunde“. Die Form $\lambda \tilde{\omega}$ bedeutet „ich will“ (...). So Philoxenos in der Abhandlung Über den ionischen Dialekt (fr. 296 Theodoridis). (Or. 95,12 Sturz) ${ }^{24}$

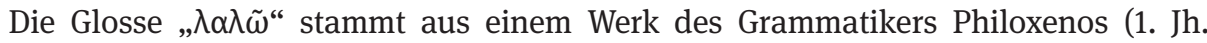
v.Chr.), der explizit als Quelle angegeben wird..$^{25}$ Der Name des Tragikers ist im Text der Manuskripte des Orionlexikons ausgelassen worden, aber es kann mithilfe der indirekten Überlieferung rekonstruiert werden, dass das Zitat bei Orion ursprünglich mit dem Autorennamen verbunden war. ${ }^{26}$ So wird bestätigt, dass der hier herangezogene Vers aus Euripides stammt, und zwar aus der Andromache:

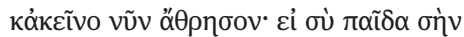

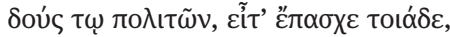

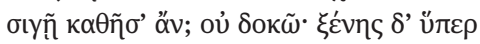

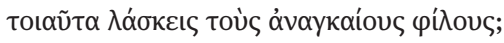

Auch das bedenke: Hättest deine Töchter du

vermählt mit einem Bürger und es ging ihr so,

bleibst du dann ruhig sitzen? Doch wohl nicht! Und um

ein fremdes Weib schmähst derart du die nächsten Freunde? (Eur. Andr. 668-671, Übs. Ebener)

Aus Philoxenos ist das Euripides-Zitat im 5. Jh. n.Chr. in das Etymologikon des Orion eingeflossen. Im gedruckten Text des Orion von Sturz ist das Zitat nicht ganz voll-

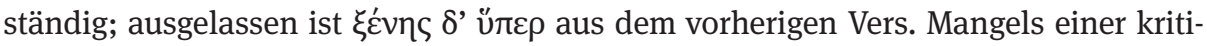
schen Ausgabe des Etymologikons lässt sich aber nicht feststellen, wann der Fehler entstanden ist. Es kommen verschiedene Erklärungen infrage: Entweder fehlten die Wörter schon bei Philoxenos, oder sie fehlten in der Kopie von Philoxenos' Werk, das Orion zugänglich war, oder Orion hat sie ausgelassen, oder sie sind innerhalb der handschriftlichen Überlieferung des Etymologikons später ausgelassen worden. Wie

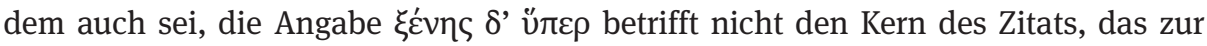
Erklärung der Verbform von $\lambda \alpha \alpha \sigma \kappa \omega$ angeführt wurde. Als nicht unmittelbar notwendig für den Kontext des Zitats konnte sie auf jeder Überlieferungsstufe leicht wegfallen bzw. weggelassen werden. ${ }^{27}$ Der Vers aus der Andromache diente schließlich nur als literarischer Beleg für eine grammatikalische Beobachtung zunächst des Philoxenos,

24 Diese Glosse lässt sich im Etymologicum Gudianum (361,31 de Stefani) und verkürzt im Etymologicum Magnum (555,36 s.v. $\lambda \alpha \lambda \tilde{\omega})$ wiederfinden.

$25 \mathrm{Zu}$ Philoxenos als Quelle des Orionslexikons vgl. Theodoridis 1976, 15-67.

26 Vgl. Theodoridis 1976, 227.

27 Vgl. Tosi 1988, 158-161. 
dann des Orion und schließlich in den byzantinischen Etymologika, die diese Glosse aus Orion übernommen haben.

\section{Euripides in der attizistischen Lexikographie}

Die Euripides-Zitate in der attizistischen Lexikographie bieten ein äußerst breites und vielschichtiges Untersuchungsgebiet. ${ }^{28}$ Korrektes Attisch zu schreiben kam im Laufe der frühen Kaiserzeit zunehmend in Mode, bis es in der zweiten Hälfte des 2. Jh. n. Chr. beinahe als notwendig für den Zugang in höhere Ämter angesehen wurde. Besonders in der Zeit Hadrians blühte der Attizismus mit den Streitigkeiten zwischen seinen Vertretern. In diesem Kontext haben attizistische Lexikographen begonnen, zielorientierte Referenzwerke $z u$ verfassen, und damit eine Tradition begründet, die sich über Jahrhunderte hinweg fortsetzte. Jeder Attizist definierte seinen eigenen Kanon der klassischen Musterautoren, die als treue Zeugen der attischen Sprache gelten sollten. Aus den unterschiedlichen Meinungen zum Kanon der zu imitierenden Autoren entstanden scharfe Polemiken und Streitigkeiten, die sich besonders in den erhaltenen Werken aus der zweiten Hälfte des 2. Jh. noch erkennen lassen. ${ }^{29}$ Außerdem ist stets $\mathrm{zu}$ bedenken, dass jedes attizistische Lexikon eine präskriptive Orientierung aufweist. Euripides und andere attische Autoren wurden herangezogen, weil sie als Maßstäbe für Stil und Wortwahl dienten.

In Bezug auf Euripides waren der Inhalt seiner Theaterstücke sowie seiner poetischen Gestaltung kaum relevant, sondern es ging vielmehr um die Frage, welche Wörter er in welcher Bedeutung benutzt hatte. Die Qualität seiner Sprache wurde außerdem an den jeweils vorherrschenden Maßstäben der attizistischen Lexikographen gemessen und gelegentlich scharf kritisiert. Um die unterschiedlichen Tendenzen der Euripides-Rezeption in der attizistischen Lexikographie zu skizzieren, seien an dieser Stelle nur wenige Beispiele kurz angeführt.

\subsection{Phrynichos}

In den auf uns gekommenen Fassungen der Werke des strengen Attizisten Phrynichos sind Euripides-Zitate selten belegt. ${ }^{30}$ In der stark verkürzten Fassung der Praeparatio sophistica ${ }^{31}$ wird Euripides einmal als positives Beispiel für die korrekte Verwendung des Adjektivs ókú $\mu \omega v$ (,steril“) herangezogen:

28 Vgl. u. a. Alpers 1990, 20 -24; Matthaios 2015, 290 mit Anm. $586 \mathrm{f}$.

29 Vgl.u. a. Lucian. Lexiph. 22; Latte 1915; Erbse 1950, 70 f.; Valente 2015, 43 f.; De Stefani 2017, 272f. mit weiterer Literatur.

30 Zu Phrynichos vgl. u. a. Kaibel 1899; Matthaios 2015, 293.

31 Nur ein alphabetisch angeordneter Auszug aus den ursprünglich 37 Büchern ist noch erhalten: vgl. von Borries 1911. 


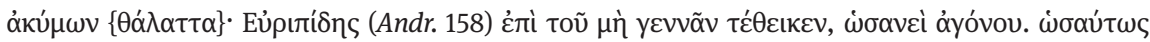

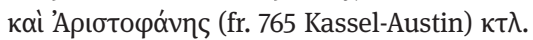

Unfruchtbar: Euripides hat es für den Begriff „nicht erzeugen“ benutzt, d.h. steril; genauso auch Aristophanes (fr. 765 Kassel-Austin) (usw.). (Phryn. Praep. soph. 6,13 von Borries)

Die hier angedeutete Euripides-Stelle stammt aus der Andromache:

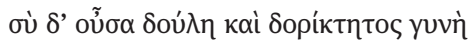

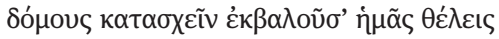

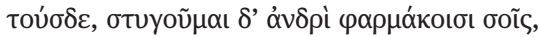

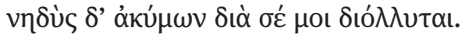

$\mathrm{Du}$, eine Sklavin nur, ein kriegsgefangenes Weib,

du willst vertreiben uns und herrschen hier im Hause!

Verhasst bin ich dem Gatten durch dein Zauberwerk,

mein Leib ist unfruchtbar, durch dich, und siecht dahin. (Eur. Andr. 155-158, Übs. Ebener)

Aufgrund der Verkürzung des Lexikons lässt sich aber nicht feststellen, ob Phrynichos tatsächlich auch den Vers der Andromache zitiert hat. Man kann nur konstatieren, dass Euripides hier gemeinsam mit Aristophanes als empfehlenswertes Beispiel herangezogen wird.

Auch in dem anderen noch erhaltenen Werk dieses Lexikographen, der Ekloge, findet sich nur ein Euripides-Zitat, erneut aus der Andromache. Die Glosse des Lexikographen betrifft das geographische Adjektiv für die Region Sparta, Lakonien:

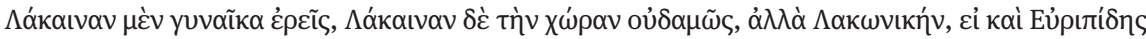

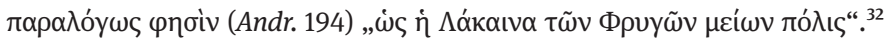

Als lakainisch wirst du eine Frau bezeichnen, keineswegs aber das Land, sondern lakonisch, auch wenn Euripides es nicht korrekt gebraucht und sagt (Andr. 194), „dass die lakainische Stadt schwächer ist als die Phrygerstadt“. (Phryn. Ecl. 318 Fischer)

Nach Phrynichos wäre also dem attischen Tragiker Euripides aus dem 5. Jh. v.Chr. ein Verstoß gegen den korrekten attischen Wortgebrauch anzulasten. In derselben Tragödie finden sich aber zwei weitere Stellen, an denen das Adjektiv benutzt wird: An der ersten wird es in der nach Phrynichos ,richtigen' Bedeutung benutzt:

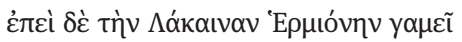

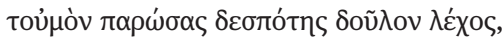

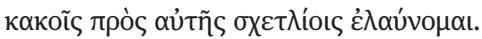

Doch seit der Herr mein Sklavenbett verschmäht und die

Spartanerin Hermione zur Gattin hat,

setzt sie mir zu mit schonungslosen Quälereien. (Eur. Andr. 29-31, Übs. Ebener)

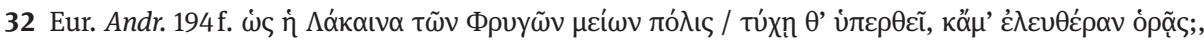
„Ist Sparta schwächer als die Phrygerstadt? Hat diese / mehr Glück? Siehst einen freien Menschen du in mir?“ (Übs. Ebener). 
An der zweiten in der nach Phrynichos ,falschen‘ Bedeutung:

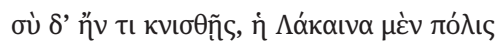

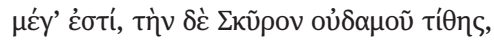

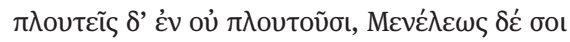

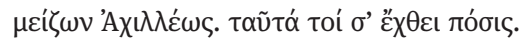

Und quält dich noch etwas, dann dieses: Groß ist Sparta, doch Skyros achtest du für nichts. Du schwelgst im Reichtum vor armen Leuten. Menelaos gilt für dich mehr als Achilleus. Darum ist dein Mann dir gram. (Eur. Andr. 209-212, Übs. Ebener)

Dass das Zitat nicht aus direkter Lektüre der Tragödie stammt, lässt sich wohl anhand eines anderen ihm gegenwärtigen Lexikons erklären. Ein polemisches Ziel des Phrynichos war unter anderen das lexikographische Werk eines für uns anonymen Gelehrten, des sogenannten Antiattizisten. ${ }^{33}$ Dieser Lexikograph war ein Vertreter eines milderen Attizismus, denn er billigte einen breiteren Kanon von Musterautoren als Phrynichos. Beispiel dafür ist auch die Glosse, auf die Phrynichos in diesem Fall vermutlich Bezug nimmt. Der Antiattizist verteidigte die umstrittene Bedeutung von $\Lambda \alpha ́ k \alpha ı v \alpha$ in Verbindung mit Ortsangaben mithilfe einer Stelle des Komikers Alexis:

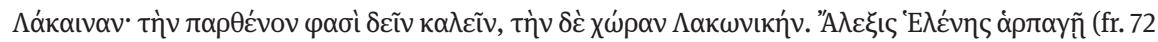
Kassel-Austin).

Lakainisch: Man sagt, man solle so eine junge Frau nennen, das Land aber lakonisch. Alexis in der Entführung der Helena (fr. 72 Kassel-Austin) benutzt aber lakainisch für das Land. (Antiatt. $\lambda 16$ Valente)

Alexis' Sprache galt aber den meisten Attizisten nicht als gutes Attisch, denn dieser Komiker gehörte zur sogenannten mittleren bzw. neuen Komödie. ${ }^{34}$ Phrynichos konnte die Stellungnahme des Antiattizisten deshalb nicht akzeptieren. Denn seine Grundüberzeugung war, dass es einen klaren Unterschied zwischen den beiden Adjektiven geben müsse. Da er in seinen Quellen aber einen Euripides-Passus fand, der die Position seines Gegners durch einen ,besseren' attischen Schriftsteller sogar noch hätte untermauern können, zog er den Schluss, dass bereits Euripides hier ein Fehler unterlaufen sei. So werden die Leser und Nutzer seiner Ekloge unmissverständlich

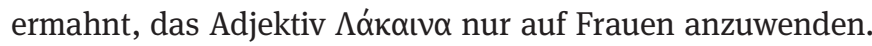

\subsection{Julius Pollux und der Antiattizist}

Eine ähnlich kritische Stellungnahme zur Sprache des Euripides findet man gelegentlich in einem anderen zentralen attizistischen Werk aus derselben Zeit. Im On-

33 Vgl. Valente 2015.

34 Vgl. u. a. Arnott 1996, 18-25, 41-43; Willi 2010, 475-477. 
omastikon des Julius Pollux, einem umfangreichen Werk in zehn Büchern, das in einer leicht verkürzten Fassung erhalten ist, werden synonyme Begriffe aneinandergereiht, um den Rednern und Schriftstellern ein möglichst umfassendes Repertorium von Wörtern für die literarische und rhetorische Komposition zu geben. ${ }^{35}$ Die Begriffe sind oft durch Beispiele aus klassischen Autoren illustriert; nicht selten spricht Pollux in erster Person, um seine Meinung noch deutlicher $\mathrm{zu}$ machen. ${ }^{36}$ Für unsere Fragestellung sei folgender Abschnitt des Onomastikons, an dem die unterschiedlichen Synonyme für die Begriffe „Herrscher“ bzw. „Herrscherin“ vorgestellt werden, beispielhaft betrachtet:

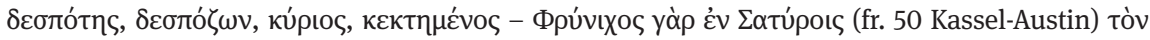

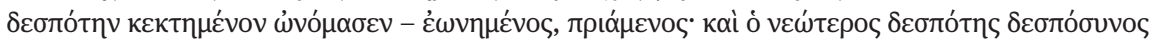

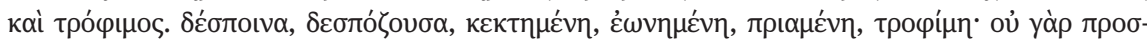

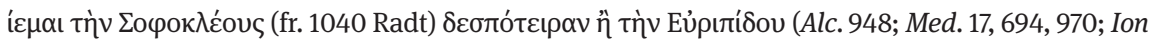

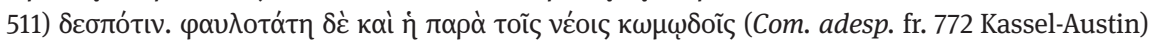

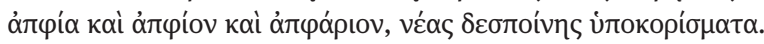

Herrscher, Beherrschender, Herr, Meister - denn Phrynichos nannte in den Satyroi (fr. 50 KasselAustin) den Herrn „Meister“ -, Eigentümer, Besitzer; und der junge Herrscher junger Herr und Hausherr. Herrscherin, Beherrschende, Meisterin, Eigentümerin, Besitzerin, junge Meisterin.

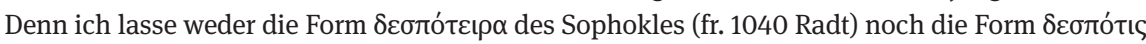

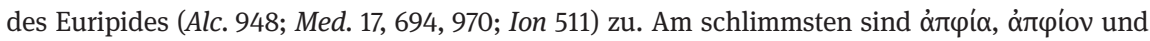

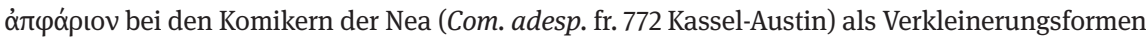
für eine junge Herrscherin. (Poll. 3,73f. Bethe) ${ }^{37}$

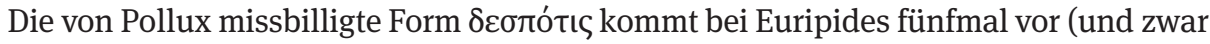
in der Alkestis, in der Medea und im Ion), selbstverständlich unterschiedlich dekliniert. Ob der Lexikograph auf eine genaue Textstelle aus einer dieser Tragödien oder aus einem heute verschollenen Werk des Euripides verweisen wollte, kann aber aufgrund der im Onomastikon wohl verkürzten Angabe nicht genauer identifiziert werden.

Um die Perspektive der Attizisten noch deutlicher zu schildern, lässt sich eine weitere Glosse des Antiattizisten anführen:

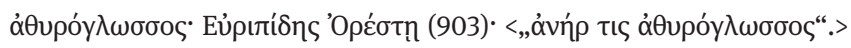

Schwätzer: Euripides im Orestes (903): „ein schwätzender Mann“. (Antiatt. $\alpha 61$ Valente)

Der Antiattizist billigte also das selten gebrauchte Adjektiv å $\theta v \rho o ́ y \lambda \omega \sigma \sigma o \varsigma$ anhand eines Zitats aus dem Orestes:

35 Vgl. Matthaios 2015, 294 mit Literatur.

36 Vgl. Matthaios 2013; Valente 2013.

37 Vgl. auch Antiatt. к 31 Valente; Eust. In Il. 565,23 und 971,33 (Ar. Byz. 80 Slater). 


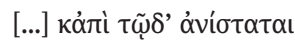

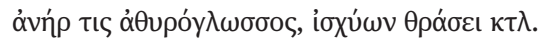

[...] Nach diesem kam zu Wort

ein Schwätzer, einflussreich durch Dreistigkeit (...). (Eur. Or. 902f., Übs. Ebener)

Im zweiten Buch seines Onomastikons behandelt Pollux die Komposita des Wortes y $\lambda \tilde{\omega} \tau \tau \alpha$. Derselbe Euripides-Passus wird hier aufgegriffen:

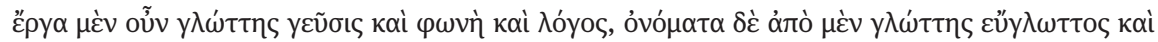

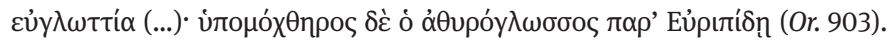

Die Funktionen der Zunge sind das Kosten, die Stimme und die Rede; Wörter, die von dem Wort „Zunge“ abgeleitet werden sind „beredt“ („zungenfertig“) und „Beredsamkeit“ (...); stilistisch schlecht ist das Wort „Schwätzer“ („einer, der seine Zunge nicht im Zaum halten kann“) bei Euripides (Or. 903). (Poll. 2,108f. Bethe)

Das Urteil des Lexikographen ist an dieser Stelle unmissverständlich. Dadurch lässt sich noch einmal belegen, mit welcher Perspektive sich die Attizisten des Euripides bedienten: Sein Sprachgebrauch diente hauptsächlich als Repertorium für die zeitgenössische literarische und rhetorische Produktion; wo immer Euripides nicht im Einklang mit der Idee attischer - d.h. hier attizistischer - Sprache erschien, musste der Dichter falsch liegen oder sich einer schlechten Ausdrucksweise bedient haben. Auch bei den Attizisten beschränkt sich also die Euripides-Rezeption fast nur auf seine Sprache und Wortwahl, die wohl meistens durch gelehrte Zwischenquellen weitergegeben und auf diese Weise betrachtet und beurteilt wurde.

\section{Fazit}

Anhand der besprochenen Textstellen lässt sich folgendes Fazit ziehen: In der Lexikographie aus der Kaiserzeit und der Spätantike scheint Euripides meistens aus zweiter bzw. dritter Hand rezipiert worden zu sein. Zitate aus seinen Dramen werden in der Regel nicht direkt aus einer aktiven Lektüre seiner Dramen, sondern vielmehr aus lexikographischen und grammatikalischen Repertorien aus hellenistischer und frühkaiserlicher Zeit, insbesondere aus den Werken des Didymos übernommen. Diese gelehrten Materialien wurden überarbeitet und nach Bedarf in die neuen Lexika eingefügt: Euripides-Zitate dienten als literarische Belege für die Erklärung eines Begriffs bzw. einer Redewendung, sei es aus historisch-antiquarischen Interessen, sei es als Beleg für grammatikalische, semantische oder syntaktische Eigenschaften.

In der attizistischen Lexikographie nimmt die Euripides-Rezeption eine besondere Stellung ein, denn seine Sprache wird oft als Quelle für Ausnahmen bzw. Besonderheiten herangezogen. Diejenigen Attizisten, die eine strenge Haltung bezüglich des Kanons der mustergültigen Autoren zeigen, tendieren in der Regel dazu, Euripides’ Sprache abzulehnen. In den attizistischen Lexika lassen sich gegensätzliche und 
zum Teil tendenziöse Beurteilungen der Ausdrucksweise und Wortwahl des Euripides entdecken, die noch genauer zu untersuchen sind. Zu diesem Zweck ist es notwendig, jedes einzelne Zitat zunächst für sich, sodann im Kontext des gesamten Werkes und zuletzt im Vergleich mit der noch erhaltenen weiteren lexikographischen Produktion zu untersuchen, um Fehlinterpretationen zu vermeiden.

\section{Literatur:}

Alpers 1990: Klaus Alpers, „Griechische Lexikographie in Antike und Mittelalter. Dargestellt an ausgewählten Beispielen“, in Hans-Albert Koch (Hg.), Welt der Information. Wissen und Wissensvermittlung in Geschichte und Gegenwart, Stuttgart, 14-38.

Alpers 2005: Klaus Alpers, „Corrigenda et Addenda to Latte's Prolegomena to Hesychii Alexandrini Lexicon Vol. I: A- $\Delta$ “, in: Peter Allan Hansen (Hg.), Hesychii Alexandrini Lexicon, III, Berlin/New York 2005, XV-XXIII.

Arnott 1996: William Geoffrey Arnott (Hg.), Alexis: The Fragments. A Commentary, Cambridge.

Barrett 1964: William Spencer Barrett (Hg.), Euripides. Hippolytos, Oxford.

Biehl 1970: Werner Biehl (Hg.), Euripides. Troades, Leipzig.

Biehl 1989: Werner Biehl (Hg.), Euripides. Troades, Heidelberg.

Billerbeck u. Neumann-Hartmann 2017: Margarethe Billerbeck u. Arlette Neumann-Hartmann (Hgg.), Stephani Byzantii Ethnika, V, Berlin/Boston.

Braswell 2013: Bruce K. Braswell, Didymos of Alexandria. Commentary on Pindar. Edited and Translated with Introduction, Explanatory Notes, and a Critical Catalogue of Didymus' Works, Basel.

Cunningham 2009: Ian C. Cunnigham, „Prolegomena“, in: Peter A. Hansen u. Ian C. Cunningham (Hgg.), Hesychii Alexandrini Lexicon, IV, Berlin/New York 2009, XI-XIX.

De Stefani 2017: Claudio De Stefani, Rez. zu Valente 2015, Aiônos. Miscellanea di Studi Storici 21, 267-273.

Dunbar 1995: Nan Dunbar (Hg.), Aristophanes. Birds, Oxford.

Erbse 1950: Hartmut Erbse, Untersuchungen zu den attizistischen Lexika, Berlin.

Fraenkel 1950: Eduard Fraenkel (Hg.), Aeschylus. Agamemnon, I-III, Oxford.

Kaibel 1899: Georg Kaibel, De Phrynicho sophista, Göttingen.

Kannicht 1969: Richard Kannicht (Hg.), Euripides. Helena, I-II, Heidelberg.

Kannicht 2004: Richard Kannicht (Hg.), Tragicorum Graecorum fragmenta (TrGF), 5.1-2: Euripides, Göttingen.

Latte 1915: „Zur Zeitbestimmung des Antiatticista“, Hermes 50 (1915) 373-394 (= ders., Kleine Schriften zu Religion, Recht, Literatur und Sprache der Griechen und Römer, hg.v. Olof Gigon, Wolfgang Buchwald u. Wolfgang Kunkel, München 1968, 612-630).

Latte 1953: Kurt Latte (Hg.), Hesychii Alexandrini Lexicon, I, Kopenhagen.

Latte-Cunningham 2018: Hesychii Alexandrini Lexicon, I, recensuit et emendavit Kurt Latte, editionem alteram curavit lan C. Cunningham, Berlin/Boston.

Matthaios 2013: Stephanos Matthaios, „Pollux’ Onomastikon im Kontext der attizistischen Lexikographie. Gruppen ,anonymer Sprecher' und ihre Stellung in der Sprachgeschichte und Stilistik“, in Mauduit 2013, 67-140.

Matthaios 2015: Stephanos Matthaios, „Philology and Grammar in the Imperial Era and Late Antiquity. An Historical and Systematic Outline“, in: Franco Montanari, Stephanos Matthaios u. Antonio Rengakos (Hgg.), Brill's Companion to Ancient Greek Scholarship, Leiden/Boston, 184-296. 
Mauduit 2013: Christine Mauduit (Hg.), L'Onomasticon de Pollux: aspects culturels, rhétoriques et lexicographiques, Lyon.

Reitzenstein 1897: Richard Reitzenstein, Geschichte der griechischen Etymologika. Ein Beitrag zur Geschichte der Philologie in Alexandria und Byzanz, Leipzig.

Schmidt 1854: Moritz Schmidt (Hg.), Didymi Chalcenteri Grammatici Alexandrini fragmenta quae supersunt omnia, Leipzig 1854.

Schmidt 1868: Moritz Schmidt (Hg.), Hesychii Alexandrini Lexicon, V, Jena 1868.

Theodoridis 1976: Christos Theodoridis (Hg.), Die Fragmente des Grammatikers Philoxenos, Berlin/New York.

Theodoridis 1982: Christos Theodoridis (Hg.), Photii Patriarchae Lexicon, I, Berlin/New York.

Tosi 1988: Renzo Tosi, Studi sulla tradizione indiretta dei classici greci, Bologna.

Valente 2018: Stefano Valente, „Hesychius“, in: Franco Montanari, Fausto Montana u. Lara Pagani (Hgg.), Brill's Lexicon of Greek Grammarians of Antiquity (LGGA), Leiden/Boston (<https:// referenceworks.brillonline.com/browse/lexicon-of-greek-grammarians-of-antiquity/ *Hesychius $)$.

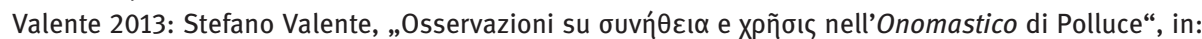
Mauduit 2013, 147-163.

Valente 2015: Stefano Valente (Hg.), The Antiatticist. Introduction and Critical Edition, Berlin/Boston.

von Borries 1911: Johann von Borries (Hg.), Phrynichi Sophistae Praeparatio sophistica, Leipzig. Wilamowitz ${ }^{3}$ 1921: Ulrich von Wilamowitz-Moellendorff, Einleitung in die griechische Tragödie, Berlin.

Willi 2010: Andreas Willi, The Language of Greek Comedy“, in Gregory W. Dobrov (Hg.), Brill's Companion to the Study of Greek Comedy, Leiden/Boston 2010, 471-510. 
